\title{
IMR
}

Letter to the Editor

\section{"Drosha, DGCR8, and Dicer mRNAs are downregulated in human cells infected with dengue virus 4" - Genet. Mol. Res. 15 (2): gmr.15027891 - Drosha, Dicer, and TRBP mRNA are downregulated in Vero cells with the 3'UTR of Dengue virus}

\author{
J.A. Castillo and S. Urcuqui-Inchima \\ Grupo Inmunovirología, Facultad de Medicina, Universidad de Antioquia, \\ Medellín, Colombia \\ Corresponding author: S. Urcuqui-Inchima \\ E-mail: Silvio.urcuqui@udea.edu.co \\ Genet. Mol. Res. 15 (3): gmr. 15039050 \\ Received August 2, 2016 \\ Accepted August 19, 2016 \\ Published August 30, 2016 \\ DOI http://doi.dx.org/10.4238/gmr.15039050 \\ Copyright $(2016$ The Authors. This is an open-access article distributed under the terms of \\ the Creative Commons Attribution ShareAlike (CC BY-SA) 4.0 License.
}

Dear Editor,

A recent paper (Casseb et al., 2016) published in the journal Genetics and Molecular Research described the interesting concept that dengue virus (DENV)-4 infection, in the human cell line A-549, leads to the downregulation of expression of key components of microRNA (miRNA) biogenesis, such as Drosha, Dicer, and DGCR8. For this, the authors performed 
a time course infection of A-549 cells for 5 days. The highest viral load was observed at 3 days post-infection, which corresponded with the maximum downregulation of expression of Drosha, Dicer, and DGCR8, assayed by quantitative PCR (RT-qPCR). These results supported the recent notion of a complex interaction between DENV and the host miRNA machinery and of the host miRNA response to this particular infection. Extensive evidence has shown that DENV can take advantage of host miRNAs for its own replication (Zhu et al., 2014) and that host miRNAs can inhibit DENV replication (Wu et al., 2013).

We recently described a complex interaction between miR-133a and DENV replication (Castillo et al., 2016), in which we demonstrated that overexpression of miR-133a impairs DENV-2 replication in Vero cells. We also found that the 3'-untranslated regions (3'-UTRs) of DENV-1, -2, and -4 downregulated the expression of miR-133a. In the context of this study, our data was similar to that reported by Casseb et al. (2016), indicating that transfection of the 3'-UTRs of DENV-1, -2, and -4 into Vero cells induced downregulation of the expressions of Drosha, Dicer, and TAR RNA-binding protein (TRBP), all of which are key components of the miRNA biogenesis machinery. The detailed methods for obtaining the 3'-UTRs of DENV$1,-2$, and -4 fused with green fluorescent protein have been described previously (Castillo et al., 2016). Vero cells were transfected with the different 3'-UTRs and total RNA was purified. Vero cells without transfection or transfected with pEGFP-C1 empty vector were used as controls. cDNA reactions were carried out using 10 ng total RNA, using the RevertAid Minus First Strand cDNA Synthesis Kit (Thermo Fisher Scientific, USA). qPCR was performed in triplicates using the SYBR green PCR master mix (Thermo Fisher Scientific), with a set of primers specific for Drosha, Dicer, and TRBP, as previously described (Sanghvi and Steel, 2011; Ren et al., 2012). Ct values were calculated for each reaction and were normalized to uninfected control and 18S rRNA $(\Delta \Delta \mathrm{Ct})$ to obtain the fold change. As seen in Figure 1, the 3'-UTRs of the three DENV serotypes evaluated significantly downregulated the expression levels of the three components of miRNA biogenesis (Figure 1A, B, and C). Dicer mRNA exhibited a low expression level starting at $12 \mathrm{~h}$ post-transfection (hpt), but only with the 3'-UTRs of DENV-2 and DENV-4 (Figure 1C). Nevertheless, at 24 and $48 \mathrm{hpt}$ the 3'-UTRs of the three DENV serotypes significantly decreased the expression level of Dicer mRNA (Figure 1C), with the lowest level at $24 \mathrm{hpt}$ with all 3'-UTRs ( 2.3 folds). The 3'-UTRs of the three DENV serotypes also significantly decreased Drosha mRNA expression at $72 \mathrm{hpt}$ ( $\sim 1.5$ fold; Figure 1A). The TRBP mRNA expression level was also affected by the 3'-UTRs of the three DENV serotypes but at different times during evaluation (Figure 1B). It should be noted that the 3'-UTR of DENV-2 induced a significant downregulation of the mRNAs of all the evaluated proteins of the miRNA machinery, with the highest effect at $72 \mathrm{hpt}$ for TRBP ( $\sim 6$ folds; Figure 1D). Taken together, these results show that the 3'-UTRs of the three DENV serotypes can significantly alter the expression of the miRNA biogenesis proteins.

These results strongly correlate with those of Casseb et al. (2016), who presented data for DENV-4 infection. Taken together, these results suggest a role of DENV, possibly through the 3'-UTR, in the regulation of cellular miRNA expression. It has been reported that other components, such as the DENV NS4B protein, directly bind Dicer and suppress its activity, affecting the production of cellular miRNAs (Kakumani et al., 2013). Similarly, the West Nile virus subgenomic flavivirus RNA inhibits cleavage of double-stranded RNA by Dicer, in vitro (Schnettler et al., 2012). Although these are among the first reports showing a link between DENV infection and the host miRNA machinery, a growing number of studies have reported the functions of miRNA/RNA interference machinery in DENV replication (Sánchez-Vargas

Genetics and Molecular Research 15 (3): gmr.15039050 
et al., 2009; Hess et al., 2011). These data support the idea that noncoding sequences of DENV, such as the 3'-UTR, might be involved in host miRNA inhibition.
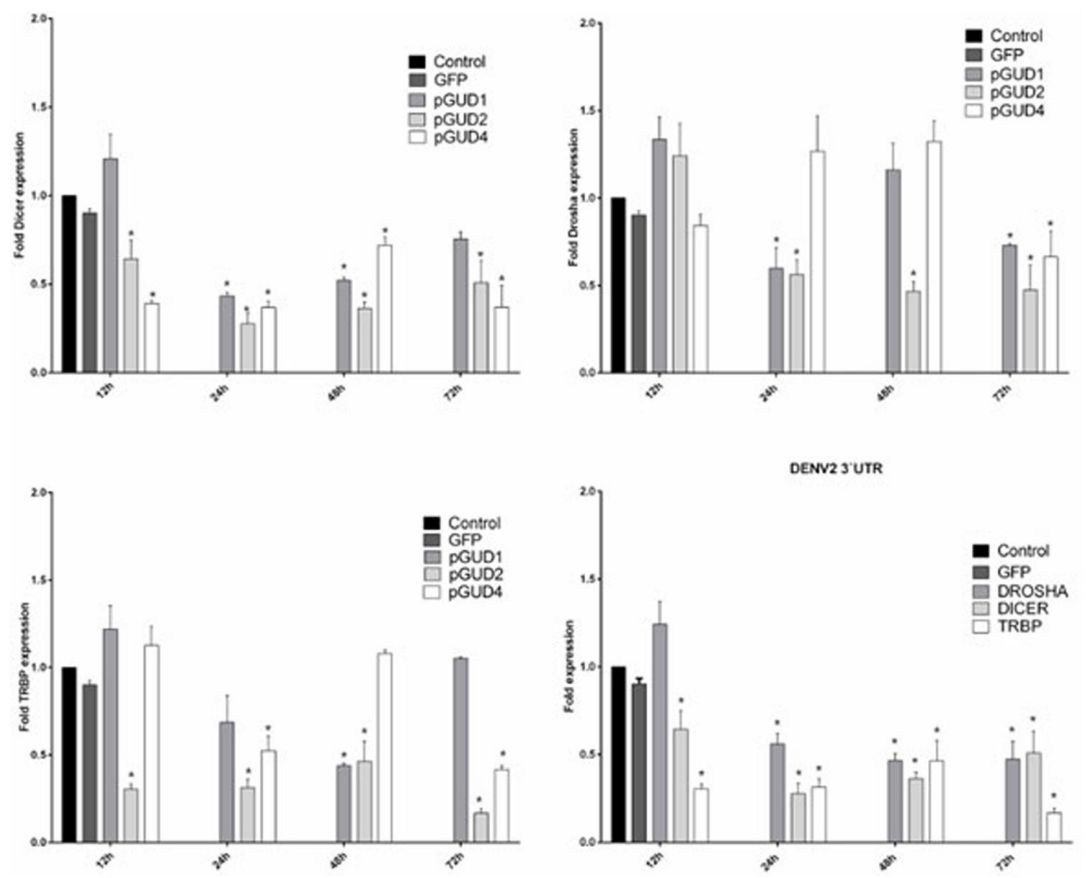

Figure 1. Transfection of Dengue virus (DENV) 3'-untranslated regions (UTRs) modulates the expression of mRNAs of the components of miRNA pathway. Vero cells were transfected with pEGFP plasmids carrying the 3'-UTRs of DENV-1 (D1 3'-UTR), -2 (D2 3'-UTR), or -4 (D4 3'-UTR). Then, Drosha (A), TRBP (B), and Dicer (C) mRNA expressions were evaluated at $12,24,48$, and $72 \mathrm{~h}$ post-transfection by real-time PCR. The expressions were normalized to an untransfected control and to $18 \mathrm{~S}$ rRNA $(\Delta \Delta \mathrm{Ct})$. D. This represents the data of only the DENV-2 3'-UTR. Data are reported as median \pm range of at least three separate experiments. ${ }^{*} \mathrm{P}<0.05$, compared to the control, using two-way ANOVA. TRBP, TAR RNA-binding protein; GFP, green fluorescent protein.

In conclusion, more detailed and deep studies on the interaction between DENV replication and host miRNA machinery and the response of host miRNAs need to be conducted. Much evidence suggests that various miRNAs can inhibit DENV replication, supporting the notion that DENV inhibits miRNA biogenesis (e.g., Dicer, Drosha, TRBP, and DGCR8). However, this should be analyzed in detail, because not all miRNAs are downregulated, some are even upregulated, during DENV infection (Wu et al., 2013). Moreover, different responses could be generated, depending on the time point post-infection. Nevertheless, the possibility remains that at the peak point of infection, DENV can inhibit host miRNA biogenesis.

\section{ACKNOWLEDGMENTS}

The authors thank Anne-Lise Haenni for reading the manuscript and for her constructive and valuable comments. This work was supported by Colciencias, Colombia (grant No. 111551928777) and Universidad de Antioquia, CODI (mediana cuantía, 2011) acta 624. The funders had no input in the study design.

Genetics and Molecular Research 15 (3): gmr.15039050 


\section{REFERENCES}

Casseb SM, Simith DB, Melo KF, Mendoca MH, et al. (2016). Drosha, DGCR8, and Dicer mRNAs are down-regulated in human cells infected with dengue virus 4, and play a role in viral pathogenesis. Genet. Mol. Res. 15: gmr.15027891.

Castillo JA, Castrillón JC, Diosa-Toro M, Betancur JG, et al. (2016). Complex interaction between dengue virus replication and expression of miRNA-133a. BMC Infect. Dis. 16: 29. http://dx.doi.org/10.1186/s12879-016-1364-y

Hess AM, Prasad AN, Ptitsyn A, Ebel GD, et al. (2011). Small RNA profiling of Dengue virus-mosquito interactions implicates the PIWI RNA pathway in anti-viral defense. BMC Microbiol. 11: 45. http://dx.doi.org/10.1186/14712180-11-45

Kakumani PK, Ponia SS, S RK, Sood V, et al. (2013). Role of RNA interference (RNAi) in dengue virus replication and identification of NS4B as an RNAi suppressor. J. Virol. 87: 8870-8883. http://dx.doi.org/10.1128/JVI.02774-12

Ren M, Qin D, Li K, Qu J, et al. (2012). Correlation between hepatitis B virus protein and microRNA processor Drosha in cells expressing HBV. Antiviral Res. 94: 225-231.http://dx.doi.org/10.1016/j.antiviral.2012.04.004

Sánchez-Vargas I, Scott JC, Poole-Smith BK, Franz AW, et al. (2009). Dengue virus type 2 infections of Aedes aegypti are modulated by the mosquito's RNA interference pathway. PLoS Pathog. 5: e1000299. http://dx.doi.org/10.1371/ journal.ppat.1000299

Sanghvi VR and Steel LF (2011). The cellular TAR RNA binding protein, TRBP, promotes HIV-1 replication primarily by inhibiting the activation of double-stranded RNA-dependent kinase PKR. J. Virol. 85: 12614-12621. http://dx.doi. org/10.1128/JVI.05240-11

Schnettler E, Sterken MG, Leung JY, Metz SW, et al. (2012). Noncoding flavivirus RNA displays RNA interference suppressor activity in insect and Mammalian cells. J. Virol. 86: 13486-13500. http://dx.doi.org/10.1128/JVI.01104-12

Wu S, He L, Li Y, Wang T, et al. (2013). miR-146a facilitates replication of dengue virus by dampening interferon induction by targeting TRAF6. J. Infect. 67: 329-341. http://dx.doi.org/10.1016/j.jinf.2013.05.003

Zhu X, He Z, Hu Y, Wen W, et al. (2014). MicroRNA-30e* suppresses dengue virus replication by promoting NF-kBdependent IFN production. PLoS Negl. Trop. Dis. 8: e3088.http://dx.doi.org/10.1371/journal.pntd.0003088

Genetics and Molecular Research 15 (3): gmr.15039050 